Research in Economics (1999) 53, 77-99

Article No. reec.1998.0183

Available online at http://www.idealibrary.com on IDE I

\title{
Synchronic information, knowledge and common knowledge in extensive games
}

\author{
Pierpaolo BatTigalli $\dagger$ and Giacomo Bonanno $\ddagger$ \\ $\dagger$ Department of Economics, European University Institute, \\ I-50016 Fiesole (FI), Italy and Department of Economics, \\ Princeton University, Princeton, NJ 08544-1021, U.S.A. \\ $\ddagger$ Department of Economics, University of California, \\ Davis, CA 95616-8578, U.S.A.
}

(Received March 1998, accepted June 1998)

\begin{abstract}
Summary
Restricting attention to the class of extensive games defined by von Neumann and Morgenstern (1944) with the added assumption of perfect recall, we specify the information of each player at each node of the game-tree in a way which is coherent with the original information structure of the extensive form. We show that this approach provides a framework for a formal and rigorous treatment of questions of knowledge and common knowledge at every node of the tree. We construct a particular information partition for each player and show that it captures the notion of maximum information in the sense that it is the finest within the class of information partitions that satisfy four natural properties. Using this notion of "maximum information" we are able to provide an alternative characterization of the meet of the information partitions.
\end{abstract}

J.E.L. Classification: C70, C72.

Keywords: Information partition, knowledge, common knowledge, extensive game.

\section{Introduction}

The language of extensive games is complex and rich. It allows one to express such notions as the order of moves, the information a player has when it is her turn to move, etc. It is not, however, a sufficiently rich language in the sense that there are meaningful

$\dagger$ E-mail: battigal@iue.it

† E-mail: gfbonanno@ucdavis.edu

$1090-9443 / 99 / 010077+23 \$ 30.00 / 0$ 


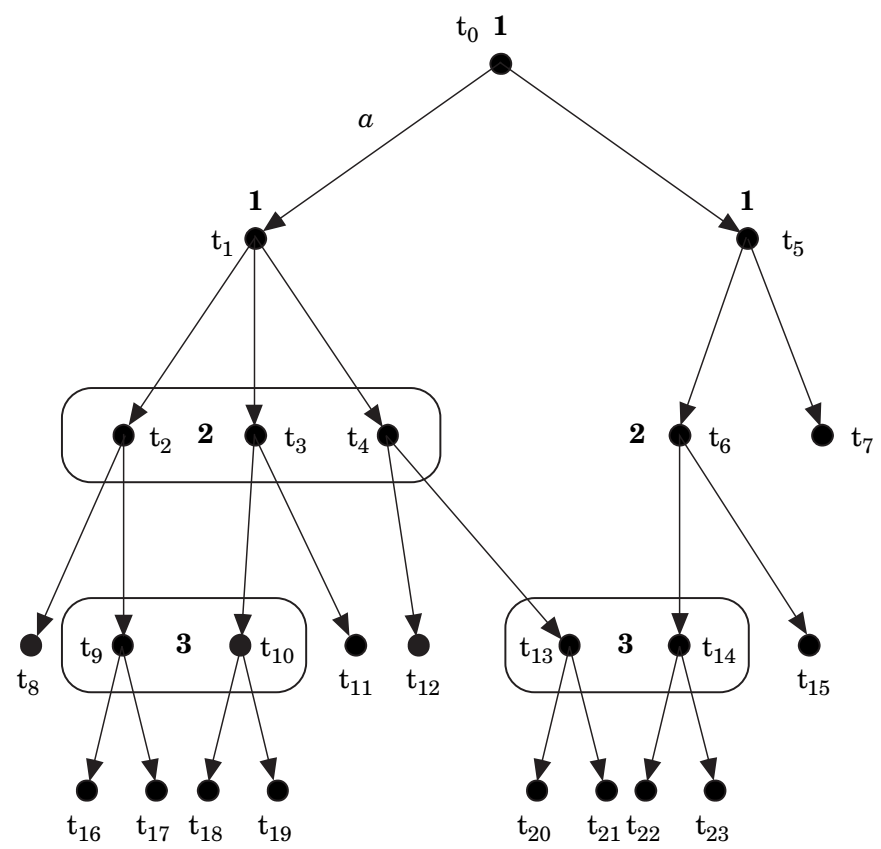

FiguRe A.

and natural statements that one can make (about a given extensive game) whose truth cannot be decided without making the language richer. We shall give two examples. Consider first the extensive form of Figure A.

Consider the following statement:

If node $t_{2}$ is reached, then every player knows that player 1 chose action $a$, but it is not common knowledge among the players that player 1 chose $a$.

Is this statement true for the extensive game of Figure A? If one adopts the standard semantics for knowledge and common knowledge $\dagger$ then, in order to answer this question, one needs the following:

(1) a set of "states" (or "possible worlds"), $\Omega$;

(2) to interpret the sentences "node $t_{2}$ is reached" and "player 1 chose $a$ " as events, that is, as subsets of $\Omega$, call them $\tau$ and $A$, respectively,

(3) for every player $i$, a partition $\mathcal{K}_{i}$ of $\Omega$ (i's information partition).

$\dagger$ See, for example, Aumann (1976), Aumann and Brandenburger (1995), Bacharach (1985), Bonanno (1996), Geanakoplos (1992), Halpern (1986), Halpern and Moses (1992), Lismont (1993), Lismont and Mongin (1994), Milgrom (1981). (This is not meant to be an exhaustive list of relevant references.) 
Once we have these three elements, deciding whether or not the above sentence is true becomes a simple matter of computation. In order for it to be true we need that:

(i) for every player $i, \tau \subseteq K_{i} A$ (where $K_{i} A$ is the event that player $i$ knows $A$ ),

(ii) $\tau \not \subset C K A$ (where $C K A$ is the event that $A$ is common knowledge).

We can, trivially, construct a model where the above sentence is true and one where it is not:

\section{VERIFYING model}

$\Omega=\{\alpha, \beta, \gamma\}$,

"node $t_{2}$ is reached" $=\tau=\{\alpha\}$

"player 1 chose $a "=A=\{\alpha, \beta\}$

$\mathcal{K}_{1}=\mathcal{K}_{2}=\{\{\alpha\},\{\beta, \gamma\}\}$,

$\mathcal{K}_{3}=\{\{\alpha, \beta\},\{\gamma\}\}$

Meet of $\mathcal{K}_{i}$ 's $=\{\{\alpha, \beta, \gamma\}\}$. Thus

$K_{1} A=K_{2} A=\{\alpha\}, K_{3} A=\{\alpha, \beta\}$,

$C K A=\phi$

\section{REFUTING model}

$\Omega=\{\alpha\}$,

"node $t_{2}$ is reached" $=\tau=\{\alpha\}$

"player 1 chose $a "=A=\phi$

This is not, however, a satisfactory answer to the above question. Both of these models are artificial, in the sense that there is nothing in them that tells us that we are talking about the extensive form of Figure A. There is no connection between the extensive form and the proposed model. On the other hand, it is quite natural to consider a model where $\Omega$ is the set of nodes of the game tree $\left(\Omega=\left\{t_{1}, t_{2}, \ldots, t_{23}\right\}=T\right)$ and those two sentences have the following interpretation:

SENTENCE

"node $t_{2}$ is reached" $\tau=\left\{t_{2}\right\}$

"player 1 chose $a$ "

\section{INTERPRETING EVENT}

$$
\begin{aligned}
A= & \left\{t_{1}, t_{2}, t_{3}, t_{4}, t_{8}, t_{9}, t_{10}, t_{11}, t_{12}, t_{13}, t_{16},\right. \\
& \left.t_{17}, t_{18}, t_{19}, t_{20}, t_{21}\right\}
\end{aligned}
$$

(set of successors of the root following $\operatorname{arc} a$ )

However, in order to analyse epistemic statements one would also need, for every player, a partition of the set of nodes $T$. The definition of extensive game does not provide us with such partitions: for every player, we are only given her information at her decision nodes and not at any other node.

As a further example of the fact that the language of extensive games is not sufficiently rich, consider the following statement:

If a node of a subgame is reached, then every player knows (or the stronger claim "it is common knowledge") that the subgame has been reached. 
Is this statement true in an arbitrary extensive game? Is it necessarily true? Again, it seems that a natural model in which to analyse such a statement would be one where the set of states is the set of nodes. Once again we are led to the issue of how to obtain, for every player, a partition of the set of nodes.

We provide a simple solution to this problem. We consider the class of extensive games defined by von Neumann and Morgenstern (1944) with the added assumption of perfect recall. Following Bonanno (1992a) we specify the information of each player at each node of the game-tree in a way which is coherent with the original information structure of the extensive form. Then the multi-stage structure of this class of games allows a characterization of the notions of knowledge and common knowledge for each stage of the game. However, the specification of this extended information structure is partially arbitrary. Therefore we consider a class of extended information structures that satisfy four natural properties and in proposition 1 we provide a constructive characterization of the finest one (that is, the one which gives maximum information). It turns out that the same kind of construction also provides an alternative characterization of the meet of these finest information partitions (and, therefore, of the notion of common knowledge with maximum information: proposition 4). We also show that it is not necessarily true that at a node of a subgame it is common knowledge among the players that the subgame has been reached, although it is true for the case of maximum information. On the other hand, it is necessarily true (that is, for all extended information structures) that in a simultaneous game at every decision node there are no non-trivial events which are common knowledge among the players.

What can one expect to gain by extending the notion of information structure in extensive games? We suggest several answers.

(1) Using extended information structures it is possible to trace the evolution of a player's beliefs along every possible play of the game. One can then impose (internal and mutual) consistency properties on belief revision and use these properties to define new equilibrium concepts (see Bonanno, 1992b) or provide alternative characterizations of known solution concepts (see Bonanno, 1995).

(2) In many cases it may be reasonable to assume that the extensive game is not an exhaustive description of the strategic situation. For example, unmodelled phases of communication and/or bargaining may occur. This is the rationale for the definition of coalition-proof and renegotiation-proof equilibria. $\dagger$

$\dagger$ See Bernheim, Peleg and Whinston (1987), Benoit and Krishna (1993), Farrell and Maskin (1989) and Greenberg (1990). 
Under this interpretation a synchronic description of players' information is essential and constitutes a prerequisite for any extension of the abovementioned solution concepts to situations of dynamic strategic interaction with differential information. For example, one might impose the restriction that a coalition of players can contemplate, at node $t$, a joint deviation from a given equilibrium only if it is common knowledge among those players, at node $t$, that the joint deviation will benefit them all. Such an approach would clearly require extended information structures, without which the notion of an event being common knowledge at an arbitrary node is not well-defined.

(3) The literature on conjectural or self-confirming equilibria [Battigalli \& Guaitoli (1997), Fudenberg \& Levine (1993), Kalai \& Lehrer (1993a,b), Rubinstein \& Wolinsky (1994)] interprets the given extensive game as a constituent of a larger repeated game (with, possibly, imperfect monitoring) and tries to define and characterize outcomes that can be "stable" under plausible learning processes. In this literature, information partitions on the set of terminal nodes of the game are taken as primitive and issues of (common) knowledge are relevant. The approach put forward in this paper extends to the entire game and provides a rationale for the information partitions on the set of end nodes of the constituent game.

(4) The notion of extended information structure can considerably simplify the representation of games. Suppose, for instance, that we want to describe a parlour game as an extensive game where at the beginning a dice is thrown, every player observes the result and then, say, player 1 has to make the next move. That every player observes the outcome of a dice can be modelled in a standard extensive game by drawing after each of the six moves of Nature a sequence of information sets for each player, where each information set consists of a single node and is followed by a single action. Each of these information sets indicates that the corresponding player gets informed, not that he has to make a decision. After that we model the remaining game, starting with the information sets for player 1 . Of course, hardly anybody will do so in practice. One will not draw any information sets followed by single actions and will draw information sets for the opponents of player 1 only after player 1 has moved. But this practice does not properly reflect the fact that the information about the throw of the dice and player 1's move do not come together, but in pieces. One might argue that the only information about the play of the game which can be relevant is the one that a player has when he has to make a "real" choice. And this information is described by the information sets followed by at least two actions. However, there is at least one interesting solution 
concept which is not invariant under the addition or deletion of information sets followed by single actions. This is the notion of perfect sequential equilibrium due to Grossman and Perry (1986; on this point see Noeldeke \& van Damme, 1990). Instead of drawing information sets followed by single actions we can use the extended partitions. Then it suffices to draw, in our example, one decision node (the root) for the random move followed by six decision nodes of player 1 which are followed by his actions and corresponding decision nodes. Then the description of the remaining game follows. That, for instance, everybody is informed about the outcome of the random move is described by having the six decision nodes following the origin as singleton sets in the extended partitions. Besides saving nodes and information sets the extended partitions provide directly, for each decision node, the information each player has about the play of the game until this node is reached. The extended partitions may constitute a step towards a general, alternative way to model games in extensive form, where one is not forced to misrepresent the actual flow and timing of information.

(5) Recently, Maskin and Tirole (1994) have analysed the notion of sequential Markov equilibrium in multi-stage games with simultaneous choices at every stage. Crucial to their analysis is the notion that at every stage each player has an information partition of the histories up to that point. Our approach clearly generalizes the notion of information partition over the set of histories to a larger class of games and provides a test for checking whether any given postulated partition is consistent with the original information structure of the extensive game (in order to be, it has to be a coarsening of the maximum information extended structure that we define and characterize in Section 4).

(6) In a recent paper, Aumann (1995) models explicitly the notion of common knowledge of rationality in extensive games and studies its implications. Aumann's epistemic model refers to the planning stage (before the game is played) and is applied only to perfect information games. He suggests (Aumann, 1995:17) that his results would still hold if his epistemic model were to be extended so as to encompass the play of the game, since "when the time comes for a player to move, he certainly knows at least as much as he did when play started". The approach we put forward enables one to extend any epistemic model of a game to include the set of nodes of the game-tree. $\dagger$ Furthermore,

$\dagger$ More precisely, the set of states (or possible worlds) would be of the form $T \times S$, where $T$ is the set of nodes and $S$ is some other set. 
it allows one to remove the restriction to perfect information games (for an example see Bonanno, 1994).

(7) Finally, as shown above, without extended information structures there are meaningful statements about extensive games whose truth one cannot decide.

\section{Definition of vN-M extensive form with perfect recall}

For reasons that will be discussed later (Section 7), throughout this paper we restrict attention to the class of extensive forms $\dagger$ (with or without chance moves) defined by von Neumann and Morgenstern (1944) with the added assumption of perfect recall. We shall call them "vN-M extensive forms".

DEFINITION: an extensive form is a $v N-M$ extensive form if, whenever two nodes belong to the same information set, the number of predecessors of one is equal to the number of predecessors of the other. Formally: $\forall t, t^{\prime} \in T, H\left(t^{\prime}\right)=H(t) \Rightarrow \ell\left(t^{\prime}\right)=\ell(t)$ (where $T$ is the set of nodes of the game-tree and, for every $x \in T, H(x)$ is the information set that contains $x$ and $\ell(x)$ is the number of predecessors of $x)$.

REMARK 1: every extensive form with perfect information is a $\mathrm{vN}-\mathrm{M}$ extensive form.

REMARK 2: given an extensive form which is not a vN-M extensive form, it may be possible to transform it into one by means of an "inessential transformation". Consider, for example, the extensive form of Figure B(i). It is not a vN-M extensive form because $\ell\left(t_{2}\right)=1$ and $\ell\left(t_{3}\right)=2$ and $t_{2}$ and $t_{3}$ belong to the same information set (of player 2). However, it can be transformed into one by adding a "dummy" node and assigning to it a "dummy" player with only one choice, as shown in Figure B(ii) (the dummy node is $t_{4}$ and the dummy player is 3). The interchange of simultaneous moves (see Thompson, 1952, and Bonanno, 1992c) is another "inessential transformation" that sometimes can be used to transform an extensive form which is not a vN-M extensive form into one which is, as shown in Figure C (the extensive form of Figure C(i) is not, while that of Figure C(ii) is, a vN-M extensive form). On the other hand, Figure D shows an extensive form which is not a vN-M

\footnotetext{
$\dagger$ An extensive form is an extensive game without the pay-off functions.

$\$$ Thus if $t_{0}$ denotes the root of the tree, $\ell\left(t_{0}\right)=0$. The Appendix contains a complete list and explanation of the notation and terminology used in this paper, which are quite standard.
} 


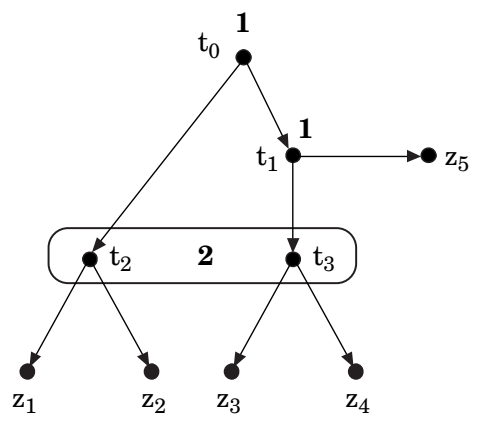

(i)

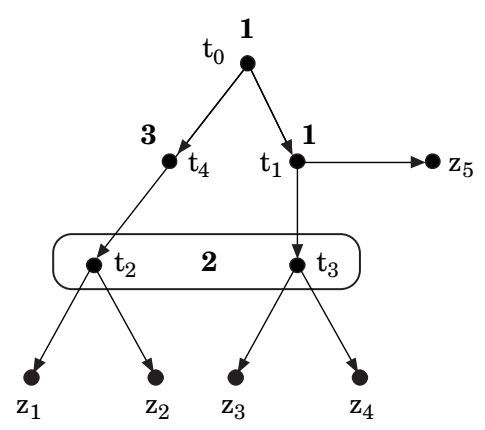

(ii)

Figure B.

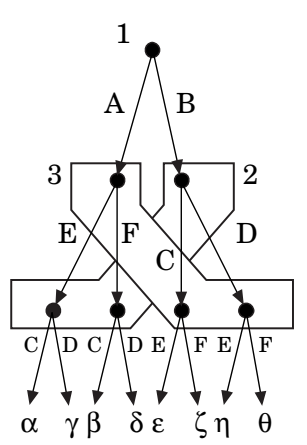

(i)

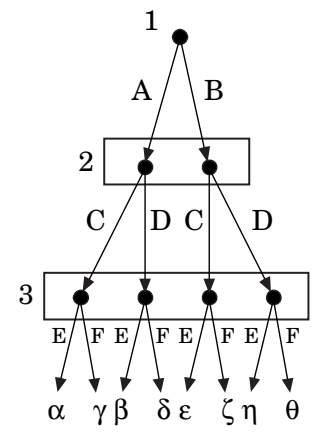

(ii)

Figure C.

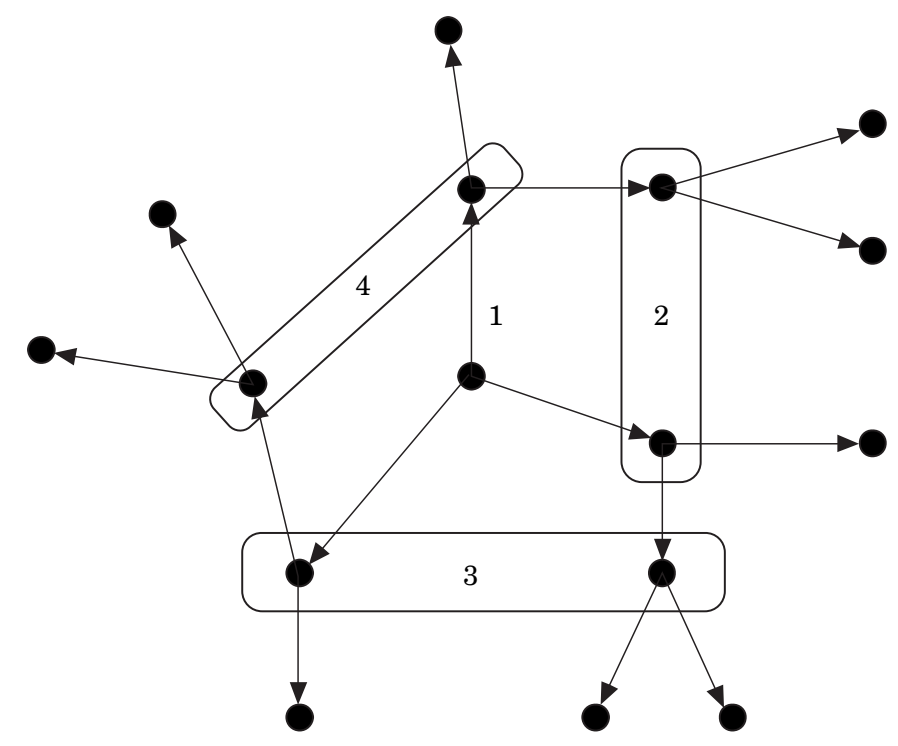

Figure D. 
extensive form and cannot be transformed into one by means of one of the transformations mentioned above.

From now on by "extensive form" we shall always mean a $v N-M$ extensive form with perfect recall.

\section{Information partitions}

As explained in the introduction, our objective is to complete the definition of extensive game by adding, for every player $i$, a partition of the set $T$ of nodes (player $i$ 's information partition). Clearly, the information structure of the game and the assumption of perfect recall impose some constraints.

Definition: let $G$ be a $v N-M$ extensive form with perfect recall. An information completion of $G$ is an $n$-tuple $\left\langle\mathcal{K}_{1}, \ldots, \mathcal{K}_{n}\right\rangle$, where, for each player $i=1, \ldots, n, \mathcal{K}_{i}$ is a partition of the set of nodes $T$, satisfying the following properties (for every node $t \in T$, we denote by $[t]_{i}$ the cell of $\mathcal{K}_{i}$ that contains $\left.t\right)$ :

(1) Coherence with the information structure $\dagger$ : if node $t$ belongs to information set $h$ of player $i$, then the cell of $\mathcal{K}_{i}$ that contains $t$ coincides with h. Formally:

$$
t \in h \in \mathbb{H}_{i} \Rightarrow[t]_{i}=h .
$$

(2) Players remember what choices they made: if the immediate predecessor of $t$ belongs to information set $h$ of player $i$ and $t$ comes after choice $c$ at $h$, then every $x \in[t]_{i}$ comes after choice $c$. Formally:

$$
(p(t) \in h) \wedge(t \in \mathcal{S}(h, c)) \Rightarrow[t]_{i} \subseteq \mathcal{S}(h, c)
$$

where $p(t)$ denotes the immediate predecessor of $t$ and $\mathcal{S}(h, c)$ the set of immediate successors of nodes in $h$ following choice $c$ at $h$.

(3) Players do not forget: if node $x$ is a successor of node $t$, then every terminal node that can be reached from a node in $[x]_{i}$ must also be reachable from some node in $[t]_{i}$ (equivalently, every node in $[x]_{i}$ must have a predecessor in $\left.[t]_{i}\right)$. Formally:

$$
t \prec x \Rightarrow\left(\forall x^{\prime} \in[x]_{i}, \exists t^{\prime} \in[t]_{i}: t^{\prime} \prec x^{\prime}\right)
$$

where $\prec$ denotes the precedence relation.

$\dagger$ Recall that, according to the definition of extensive game (see, for example, Selten, 1975), the set of decision nodes of player $i$ is partitioned into information sets (of player $i$ ). Throughout this paper we use the expression "information set" to refer to these objects. On the other hand, the elements of $\mathcal{K}_{i}$ (among which are $i$ 's information sets) will always be called cells. 
(4) Players know the stage of the game: for every node $t$, the cell containing $t$ is a subset of the set of nodes that belong to the same stage as t. Formally:

$$
\ell(t)=k \Rightarrow[t]_{i} \subseteq T^{k}
$$

(Recall that $\mathcal{\ell}(t)$ denotes the number of predecessors of $t$, that $i s$, the stage to which $t$ belongs; $T^{k}$ is the set of stage- $k$ nodes: $T^{k}=\{t \in T \mid \ell(t)=k\}$.)

By (1), $\mathcal{K}_{i} \supseteq \mathbb{W}_{i}$ (where $\mathbb{U}_{i}$ is the set of player $i$ 's information sets). Thus the elements of $\mathcal{K}_{i}$ represent a generalization of the notion of information set. Properties (2) and (3) are natural restrictions in view of the fact that we only consider extensive forms with perfect recall. Property (4) is a reflection of the multi-stage structure of the game (it is an immediate consequence of the definition of $\mathrm{vN}-\mathrm{M}$ extensive game that when player $i$ has to move she knows the stage reached by the play; condition (4) extends this property to nodes that are not $i$ 's decision nodes).

Given an extensive form $G$, in general, there are several possible information completions of it. Consider, for example, the extensive form of Figure E. To simplify, restrict attention to the set D of decision nodes (the nodes labelled $t_{0}$ to $t_{6}$ ). It is easy to see that properties (1) - (4) above imply that, for any information completion $\left\langle\mathcal{K}_{1}, \mathcal{K}_{2}\right\rangle$ of this extensive form, $\mathcal{K}_{1}$ is the finest partition, that is, for every $t \in D,[t]_{1}=\{t\}$. On the other hand, for player 2 there are several possibilities. We list two below:

$\overline{\mathcal{K}}_{2}=\left\{\left\{t_{0}\right\},\left\{t_{1}, t_{2}\right\},\left\{t_{3}\right\},\left\{t_{4}\right\},\left\{t_{5}\right\},\left\{t_{6}\right\}\right\} \quad$ MAXIMUM information $\underline{\mathcal{K}}_{2}=\left\{\left\{t_{0}\right\},\left\{t_{1}, t_{2}\right\},\left\{t_{3}, t_{5}\right\},\left\{t_{4}, t_{6}\right\}\right\} \quad$ MINIMUM information

If the information transmission rule embodied in $\overline{\mathcal{K}}_{2}$ is adopted, then more information is conveyed to player 2 than in the case where the rule expressed by $\underline{\mathcal{K}}_{2}$ is followed. In other words, $\overline{\mathcal{K}}_{2}$ is a refinement of $\underline{\mathcal{K}}_{2}$ (or the latter is a coarsening of the former).

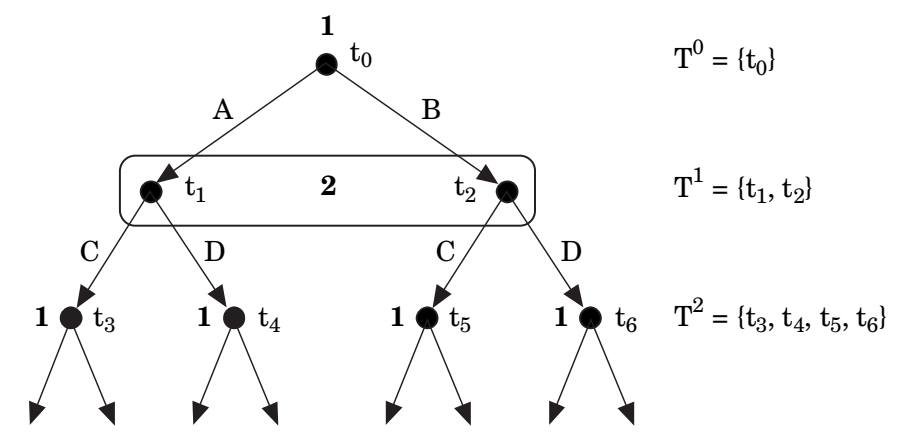

FiguRe E. 
We shall show in the next section how to generate the maximum information completion for any given extensive form.

Given an extensive form $G$ and an information completion $\left\langle\mathcal{K}_{1}, \ldots, \mathcal{K}_{n}\right\rangle$ of $G$, for every player $i$ we can define a knowledge operator $K_{i}: \wp(T) \rightarrow \wp(T)$ [where $\wp(T)$ denotes the set of subsets of $T$ ] as follows:

$$
K_{i} E=\left\{t \in T \mid[t]_{i} \subseteq E\right\} .
$$

Thus $K_{i} E$ is the event that player $i$ knows $E$. Let $\mathcal{M}$ be the meet (that is, the finest common coarsening) of the partitions $\mathcal{K}_{1}, \ldots, \mathcal{K}_{n}$. For every node $t$, denote by $[t]_{\mathcal{M}}$ the cell of $\mathcal{M}$ containing $t$. Following Aumann (1976), we define a common knowledge operator $C K: \wp(T) \rightarrow \wp(T)$ as follows:

$$
C K E=\left\{t \in T \mid[t]_{\mathcal{M}} \subseteq E\right\} .
$$

Thus $C K E$ is the event that it is common knowledge among all the players that event $E$ has occurred. $\dagger$

The extensive form of Figure $\mathrm{E}$ gives us an opportunity to answer one question raised in the introduction, namely whether it is necessarily true that if the play of the game reaches a node that belongs to subgame $G^{\prime}$, then every player knows (or the stronger claim "it is common knowledge") that $G^{\prime}$ has been reached. The answer is "No". To see this, let $G^{\prime}$ be the subgame with root $t_{3}$. Interpret the sentence "node $t_{3}$ is reached" as the event $\tau=\left\{t_{3}\right\}$ and the sentence "subgame $G^{\prime}$ has been reached" as the event $\Gamma$ consisting of $t_{3}$ and its successors. Choose the information completion where player 2's partition is given by $\underline{\mathcal{K}}_{2}$ (given above). Then $t_{3} \in K_{1} \Gamma$, but $t_{3} \notin K_{2} \Gamma$. Thus at node $t_{3}$ not every player knows (a fortiori it is not common knowledge) that $G^{\prime}$ has been reached. However, we will see later that in the case of maximum information it is indeed true, in every extensive game, that at a node of a subgame it is common knowledge among the players that the subgame has been reached.

\section{Maximum information}

In this section we construct, for every $\mathrm{vN}-\mathrm{M}$ extensive form with perfect recall, a particular information completion of it and show

$\dagger$ By property (4), for every player $i$ and every stage $k$, there is a subset of $\mathcal{K}_{i}$, call it $\mathcal{K}_{i}^{k}$, which is a partition of $T^{k}$. Thus for every $i$ and every $k$ we can define a stage- $k$ knowledge operator $K_{i}^{k}: \wp\left(T^{k}\right) \rightarrow \wp\left(T^{k}\right)$ as follows: $K_{i}^{k} E=\left\{t \in T^{k} \mid[t]_{i} \subseteq E\right\}$. Similarly, for every stage $k$ there is a subset of $\mathcal{M}$, call it $\mathcal{M}^{k}$, which is a partition of $T^{k}$. Thus we can define a stage-k common knowledge operator $C K^{k}: \wp\left(T^{k}\right) \rightarrow \wp\left(T^{k}\right)$ as follows: $C K^{k} E=\left\{t \in T^{k} \mid[t]_{M} \subseteq E\right\}$. Note, however, that for every $E \subseteq T$, $K_{i}^{k}\left(E \cap T^{k}\right)=K_{i} E \cap T^{k}$ and, similarly, $C K^{k}\left(E \cap T^{k}\right)=C K E \cap T^{k}$. 
that it is the finest (that is, the most informative) of the information completions that satisfy properties (1)-(4).

First, some notation. For every node $t$ and for every player $i$, let $\mathcal{H}_{i}(t)$ be the set of information sets of player $i$ that are crossed by paths starting at $t(t \precsim y$ means either $t=y$ or $t \prec y)$ :

$$
\mathcal{H}_{i}(t)=\left\{h \in \mathbb{U}_{i} \mid \exists y \in h: t \precsim y\right\} .
$$

Next we introduce, for every player $i$, a relation on $T$, denoted by $\sim_{i}$. Let $v, w \in T$. Then $v \sim_{i} w$ if and only if, either (i) $v=w$, or (ii) $\ell(v)=\ell(w)$ and $\mathcal{H}_{i}(v) \cap \mathcal{H}_{i}(w) \neq \phi$. The relation $\sim_{i}$ is clearly reflexive and symmetric. However, in general, it is not transitive. For example, in Figure $\mathrm{F}$ we have that $y_{1} \sim_{2} y_{2}$ and $y_{2} \sim_{2} y_{3}$ but $\operatorname{not} y_{1} \sim_{2} y_{3}$.

Let $\sim_{i}^{*}$ denote the transitive closure of $\sim_{i}$. Thus $v \sim_{i}^{*} w$ if and only if there exists a finite sequence of nodes $\left\langle y_{1}, y_{2}, \ldots, y_{m}\right\rangle$ such that $y_{1}=v, y_{m}=w$ and, for all $k=1, \ldots, m-1, y_{k} \sim_{i} y_{k+1}$. Then $\sim_{i}^{*}$ is an equivalence relation on $T$. Let $H_{i}(t)$ denote the equivalence class of $t$ generated by $\sim_{i}^{*}$ and $\overline{\mathcal{K}}_{i}$ the set of equivalence classes, that is,

$$
H_{i}(t)=\left\{v \in T^{\ell(t)} \mid v \sim_{i}^{*} t\right\}
$$

and

$$
\overline{\mathcal{K}}_{i}=\left\{A \subseteq T \mid A=H_{i}(t) \text { for some } t \in T\right\} .
$$

In lemma 1 and proposition 1 below we show that $\left\langle\overline{\mathcal{K}}_{1}, \ldots, \overline{\mathcal{K}}_{n}\right\rangle$ is an information completion [that is, it satisfies properties (1)-(4)] and that it is the finest of all the information completions that satisfy those properties. First we give an illustration based on Figures A and $\mathrm{B}(\mathrm{ii})$.

EXAMPLE 1: consider the game of Figure B(ii) where $T^{0}=\left\{t_{0}\right\}$, $T^{1}=\left\{t_{1}, t_{4}\right\}, T^{2}=\left\{t_{2}, t_{3}, z_{5}\right\}$ and $T^{3}=\left\{z_{1}, z_{2}, z_{3}, z_{4}\right\}$. Let $\overline{\mathcal{K}}_{i}^{k}$ denote the subset of $\overline{\mathcal{K}}_{i}$ that gives a partition of $T^{k}$. Then, for $i=2$ we have:

$$
\begin{aligned}
& \overline{\mathcal{K}}_{2}^{0}=\left\{H_{2}\left(t_{0}\right)=T^{0}\right\} \\
& \overline{\mathcal{K}}_{2}^{1}=\left\{H_{2}\left(t_{1}\right)=H_{2}\left(t_{4}\right)=T^{1}\right\}
\end{aligned}
$$

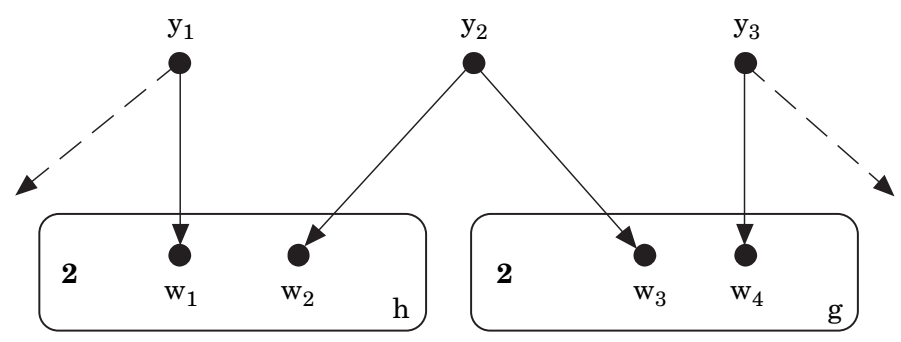

FiguRE F. 


$$
\begin{aligned}
& \overline{\mathcal{K}}_{2}^{2}=\left\{H_{2}\left(t_{2}\right)=H_{2}\left(t_{3}\right)=\left\{t_{2}, t_{3}\right\}, H_{2}\left(z_{5}\right)=\left\{z_{5}\right\}\right\} \\
& \overline{\mathcal{K}}_{2}^{3}=\left\{H_{2}\left(z_{1}\right)=\left\{z_{1}\right\}, H_{2}\left(z_{2}\right)=\left\{z_{2}\right\}, H_{2}\left(z_{3}\right)=\left\{z_{3}\right\}, H_{2}\left(z_{4}\right)=\left\{z_{4}\right\}\right\} .
\end{aligned}
$$

EXAMPLE 2: consider the game of Figure A and stage 2. Then:

$$
\begin{aligned}
& \overline{\mathcal{K}}_{1}^{2}=\left\{\left\{t_{2}\right\},\left\{t_{3}\right\},\left\{t_{4}\right\},\left\{t_{6}\right\},\left\{t_{7}\right\}\right\}, \quad \overline{\mathcal{K}}_{2}^{2}=\left\{\left\{t_{2}, t_{3}, t_{4}\right\},\left\{t_{6}\right\},\left\{t_{7}\right\}\right\} \\
& \overline{\mathcal{K}}_{3}^{2}=\left\{\left\{t_{2}, t_{3}\right\},\left\{t_{4}, t_{6}\right\},\left\{t_{7}\right\}\right\}, \text { thus the meet is } \\
& \overline{\mathcal{M}}^{2}=\left\{\left\{t_{2}, t_{3}, t_{4}, t_{6}\right\},\left\{t_{7}\right\}\right\}
\end{aligned}
$$

Using $\overline{\mathcal{K}}_{i}$ we can show that the statement (which was discussed in the Introduction) "if node $t_{2}$ is reached then every player knows that player 1 chose action $a$ but it is not common knowledge that player 1 chose $a$ " is indeed true. Interpret the sentence "node $t_{2}$ is reached" as the event $\left\{t_{2}\right\}$ and the sentence "player 1 chose action $a$ " as the event $A=$ $\left\{t_{1}, t_{2}, t_{3}, t_{4}, t_{8}, t_{9}, t_{10}, t_{11}, t_{12}, t_{13}, t_{16}, t_{17}, t_{18}, t_{19}, t_{20}, t_{21}\right\}$, that is, A consists of the successors of the root following the arc that represents choice $a$. Then we have that $K_{1} A \cap T^{2}=\left\{t_{2}, t_{3}, t_{4}\right\}=K_{2} A \cap T^{2}$ and $K_{3} A \cap T^{2}=\left\{t_{2}, t_{3}\right\}$. Since $t_{2}$ belongs to all three sets, it is indeed true that all the players know, at $t_{2}$, that player 1 chose $a$. On the other hand, $C K A \cap T^{2}=\phi$ and, therefore, also the claim that at $t_{2}$ it is not common knowledge that player 1 chose $a$ is true. This is because at $t_{2}$ player 2 considers $t_{4}$ possible and if $t_{4}$ is indeed the true "state" then player 3 considers $t_{6}$ possible and at $t_{6}$ it is not true that player 1 chose action $a$. Thus at $t_{2}$ player 2 does not know that player 3 knows that player 1 chose $a$.

REMARK 3: if $t$ is a terminal node or the root of a subgame, then for every player $i, H_{i}(t)=\{t\}$.

LEMMA 1: the information partition $\overline{\mathcal{K}}_{i}=\left\{A \subseteq T \mid A=H_{i}(t)\right.$ for some $t \in T\}$ constructed above satisfies properties (1)-(4) (cf. Section 3).

PROOF:

(1) Coherence with the information structure. Let $i \in N$ and $t \in h \in \mathbb{H}_{i}$. We want to show that $H_{i}(t)=h$. Let $k=\ell(t)$. By definition of $\mathrm{vN}-\mathrm{M}$ extensive form, $h \subseteq T^{k}$. By definition of $\sim_{i}$, $y \sim_{i} x$ for every $y, x \in h$. Thus $H_{i}(t) \supseteq h$. Suppose that $H_{i}(t) \neq h$. Then there exist a $\tau \in H_{i}(t) \backslash h$, an $x \in h$ and a $g \in \mathbb{H}_{i}$ with $g \neq h$, such that both $\tau$ and $x$ have a successor in $g$. Then the successors of $x$ in $g$ come after a choice at $h$, while the successors of $\tau$ in $g$ do not, contradicting the hypothesis of perfect recall. 
(2) Players remember what choices they made. Let $i$ be a player, $t$ a node, $h$ an information set of player $i$ and $c$ a choice at $h$. We have to show that if $p(t) \in h$ and $t \in \mathcal{S}(h, c)$ (recall that $p(t)$ denotes the immediate predecessor of $t$ and $\mathcal{S}(h, c)$ the set of immediate successors of nodes in $h$ following choice $c$ ) then $H_{i}(t) \subseteq \mathcal{S}(h, c)$. When $t$ is a decision node of player $i$, this is a trivial consequence of (1) and perfect recall. Assume, therefore, that $t$ is not a decision node of player $i$. Fix an arbitrary $\tau \in H_{i}(t)$. Then there exists a sequence $\left\langle y_{1}, \ldots, y_{m}\right\rangle$ with $y_{1}=t, y_{m}=\tau$ and $y_{k} \sim_{i} y_{k+1}$ for all $k=1, \ldots, m-1$. We prove that $\tau \in \mathcal{S}(h, c)$ by induction on $\mathrm{m}$. The statement is trivially true for $m=1$. Assume it is true for $m=r-1$. Let $\left\langle y_{1}, \ldots, y_{r-1}, y_{r}\right\rangle$ be a chain such that $y_{k} \sim_{i} y_{k+1}$ for all $k=1, \ldots, r-1$. Since $y_{r-1}$ is connected with $t$ by a chain of length $r-1$, the induction hypothesis yields $y_{r-1} \in \mathcal{S}(h, c)$. Since $y_{r-1} \sim_{i} \tau$, there is a $g \in \mathbb{U}_{i}$ which is intersected by a path from $y_{r-1}$ and a path from $\tau$. Since $y_{r-1} \in \mathcal{S}(h, c)$, by perfect recall $g$ contains only successors of nodes in $\mathcal{S}(h, c)$, therefore also $\tau \in \mathcal{S}(h, c)$.

(3) Players do not forget. Let $i \in N$ and $v, w \in T$ with $v \prec w$. We want to show that for every $w^{\prime} \in H_{i}(w)$ there exists a $v^{\prime} \in H_{i}(v)$ such that $v^{\prime} \prec w^{\prime}$. Fix an arbitrary $w^{\prime} \in H_{i}(w)$. Let $v^{\prime}$ be the unique node such that $v^{\prime} \prec w^{\prime}$ and $\ell\left(v^{\prime}\right)=\ell(v)$. Let $\left\langle w_{1}, \ldots, w_{m}\right\rangle$ be the sequence in $T^{\ell(w)}$ such that $w_{1}=w^{\prime}, w_{m}=w$ and, for all $j=1, \ldots, m-1$, $w_{j} \sim_{i} w_{j+1}$. Let $\left\langle v_{1}, \ldots, v_{m}\right\rangle$ be the corresponding sequence in $T^{\ell(v)}$ with $v_{j} \prec w_{j}$ for all $j=1, \ldots, m$ (it is possible that $v_{j}=v_{j+1}$ for some $j$; furthermore, $v_{1}=v^{\prime}$ and $\left.v_{m}=v\right)$. Note that, for every $j, \mathcal{H}_{i}\left(w_{j}\right) \subseteq \mathcal{H}_{i}\left(v_{j}\right)$ and $\mathcal{H}_{i}\left(w_{j}\right) \cap \mathcal{H}_{i}\left(w_{j+1}\right) \neq \phi$. Hence, for every $j$, $\mathcal{H}_{i}\left(v_{j}\right) \cap \mathcal{H}_{i}\left(v_{j+1}\right) \neq \phi$, that is, $v_{j} \sim_{i} v_{j+1}$. Therefore $v^{\prime} \in H_{i}(v)$.

(4) Players know the stage of the game. This is true by construction.

The following proposition shows that the information partition $\overline{\mathcal{K}}_{i}$ captures the notion of maximum information that can be conveyed to the players.

PROPOSITION 1: fix a $v N-M$ extensive form with perfect recall and a player $i$. Let $\mathcal{K}_{i}$ be an arbitrary partition of $T$ that satisfies properties (1)-(3) [a fortiori, properties (1)-(4)]. Then $\mathcal{K}_{i}$ is a coarsening of $\overline{\mathcal{K}}_{i}$, that is, for all $t \in T,[t]_{i} \supseteq H_{i}(t)$ (recall that $[t]_{i}$ denotes the cell of $\mathcal{K}_{i}$ that contains $t$, while $\bar{H}_{i}(t)$ denotes the cell of $\overline{\mathcal{K}}_{i}$ that contains $\left.t\right)$.

Proof: fix an arbitrary $t \in T$. If $H_{i}(t)=\{t\}$, there is nothing to prove. If $t \in h \in \mathbb{H}_{i}$, then by property (1), $[t]_{i}=h$ and, by lemma 1 , $H_{i}(t)=h$. Thus it only remains to consider the case where $t$ is not a decision node of player $i$ and $H_{i}(t) \neq\{t\}$. Fix an arbitrary $\tau \in H_{i}(t)$ with $\tau \neq t$. We want to show that $\tau \in[t]_{i}$. Let $k=\ell(t)$. By property (4), $[t]_{i} \subseteq T^{k}$. By definition of $H_{i}(t)$, there exists a finite sequence $y_{1}, y_{2}, \ldots, y_{m}$ in $T^{k}$ such that: $y_{1}=\tau, y_{m}=t$, and, 


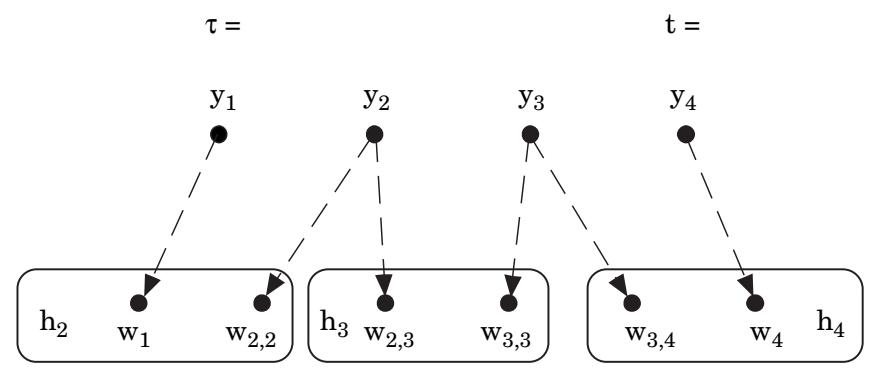

Figure G.

for every $j=2, \ldots, m$, there exists an information set $h_{j} \in \mathbb{H}_{i}$ which is crossed by a path from $y_{j-1}$ to a terminal node and by a path from $y_{j}$ to a terminal node (see Figure $\mathrm{G}$ ). Let $w_{1}$ be a successor of $y_{1}$ in $h_{2}, w_{m}$ be a successor of $y_{m}$ in $h_{m}$. For $j=2, \ldots, m-1$, let $w_{j, j}$ be a successor of $y_{j}$ in $h_{j}$ and $w_{j, j+1}$ be a successor of $y_{j}$ in $h_{j+1}$ (see Figure G). By property (1) (coherence with the information structure), $\left[w_{1}\right]_{i}=h_{2},\left[w_{m}\right]_{i}=h_{m}$, and, for every $j=2, \ldots, m-1,\left[w_{j, j}\right]_{i}=h_{j}$ and $\left[w_{j, j+1}\right]_{i}=h_{j+1}$. By property (3) (players do not forget) every node in $h_{2}$ has a predecessor in $\left[y_{1}\right]_{i}$ and a predecessor in $\left[y_{2}\right]_{i}$. Thus $\left[y_{1}\right]_{i} \cap\left[y_{2}\right]_{i} \neq \phi$. Hence $\left[y_{1}\right]_{i}=\left[y_{2}\right]_{i}$. Similarly, by property (3), every node in $h_{3}$ has a predecessor in $\left[y_{2}\right]_{i}$ and a predecessor in $\left[y_{3}\right]_{i}$. Thus $\left[y_{2}\right]_{i} \cap\left[y_{3}\right]_{i} \neq \phi$. Hence $\left[y_{2}\right]_{i}=\left[y_{3}\right]_{i}$. Repeating the same argument for $j=3, \ldots, m$, we reach the conclusion that $\left[y_{1}\right]_{i}=\left[y_{m}\right]_{i}$, that is, $[\tau]_{i}=[t]_{i}$.

From now on, in virtue of proposition 1 , we shall call $\left\langle\overline{\mathcal{K}}_{1}, \ldots, \overline{\mathcal{K}}_{n}\right\rangle$ the maximum information completion and $\overline{\mathcal{K}}_{i}$ the maximum information partition for player $i$.

We now highlight some further properties of the maximum information completion.

Proposition 2: fix a $v N-M$ extensive form with perfect recall $G$ and let $\left\langle\overline{\mathcal{K}}_{1}, \ldots, \overline{\mathcal{K}}_{n}\right\rangle$ be the maximum information completion of $G$. Then, for every node $\widehat{t}, \widehat{t}$ is the root of a subGAME if and only if, at every node $t$ such that $\widehat{t} \precsim t$, it is common knowledge among all the players that the play of the game has reached the subTREE with root $\widehat{t}$ (that is, $\widehat{t}$ is the root of a subgame if and only if, for every $t$ such that $\widehat{t} \precsim t,[t]_{\bar{M}} \subseteq\left\{v \in T^{\ell(t)} \mid \widehat{t} \precsim v\right\}$ ).

Proof: (Sufficiency). Let $\widehat{t}$ be the root of a subgame. As remarked previously, it is an immediate consequence of the definition of $H_{i}(\cdot)$ that, for every $i \in N, H_{i}(\widehat{t})=\{\widehat{t}\}$. Hence if $\overline{\mathcal{M}}$ is the meet (the finest common coarsening) of $\left\{\overline{\mathcal{K}}_{i}\right\}_{i \in N}$, then $\{\widehat{t}\} \in \overline{\mathcal{M}}$. Furthermore, if $t$ is a successor of $\widehat{t}$, then, by property (3) (players do not forget), for every player $i$, every node in $H_{i}(t)$ is a successor of $\widehat{t}$. Thus $[t]_{\overline{\mathcal{M}}}$ contains only successors of $\widehat{t}$. 
(Necessity). Suppose that $\widehat{t}$ is not the root of a subgame. Then there exist a player $i$, an $h \in \mathbb{H}_{i}$ and two nodes $w, w^{\prime} \in h$ such that $\widehat{t} \precsim w$ and $\widehat{t} \not{z} w^{\prime}$. By proposition $1, w^{\prime} \in H_{i}(w)=h$. It follows that $w^{\prime} \in[w]_{\overline{\mathcal{M}}}$. Therefore $[w]_{\overline{\mathcal{M}}} \nsubseteq\left\{v \in T^{\ell(w)} \mid \widehat{t} \precsim w\right\}$.

We showed in the preceding section (cf. Figure E) that proposition 2 is not true for a general information completion. Proposition 3 below, on the other hand, is true for all information completions.

An extensive form is said to be simultaneous if every play crosses all the information sets (thus, by perfect recall, if there are at least two choices at each information set, it must be the case that every player has exactly one information set). Let $\left\langle\mathcal{K}_{1}, \ldots, \mathcal{K}_{n}\right\rangle$ be an arbitrary information completion. Then for every decision node $t$, if $\ell(t)=k$, there must be a player $i$ and an information set $h$ of player $i$ such that $h=T^{k}$. It follows that if $\mathcal{M}$ is the meet of $\left\{\mathcal{K}_{i}\right\}_{i \in N}$, then $T^{k} \in \mathcal{M}$. Thus we have proved the following proposition.

PROPOSITION 3: fix a simultaneous game $G$, an arbitrary information completion $\left\langle\mathcal{K}_{1}, \ldots, \mathcal{K}_{n}\right\rangle$ of $G$ and a decision node t. Let $k=\ell(t)$. Then the only stage- $k$ event which is common knowledge among all the players is the certain event, that is, $T^{k}$. In other words, there are no nontrivial stage- $k$ events that are common knowledge among all the players at stage $k$.

Finally it is worth repeating that for the maximum information partition $\overline{\mathcal{K}}_{i}$ the following is true: if $z$ is a terminal node, then for every player $i, H_{i}(z)=\{z\}$. Thus, when the play of the game ends at $z$, it is common knowledge among all the players that the outcome is $z . \dagger$

\section{Maximum information and common knowledge}

In this section we show that if we restrict attention to the maximum information completion $\left\langle\overline{\mathcal{K}}_{1}, \ldots, \overline{\mathcal{K}}_{n}\right\rangle$ constructed in the previous section, then we can provide an alternative characterization of the meet $\overline{\mathcal{M}}$ of the partitions $\left\{\overline{\mathcal{K}}_{1}, \ldots, \overline{\mathcal{K}}_{n}\right\}$ (and, to this extent, of the

$\dagger$ This is the information at terminal nodes which is assumed in Fudenberg and Levine's (1993) notion of self-confirming equilibrium. In order to define more general notions of conjectural equilibria, it may be interesting to consider coarser information about terminal nodes. In particular, the one obtained by the coarsest information function, taking into account actual pay-off information (see Battigalli, 1987). Furthermore, our analysis can easily be modified in order to take into account given information partitions on the set of terminal nodes (of course, the corresponding maximum information completion would not necessarily satisfy the property about subgames stated in proposition 2 ). 
notion of common knowledge). Recall that, given an information completion $\left\langle\mathcal{K}_{1}, \ldots, \mathcal{K}_{n}\right\rangle$ (not necessarily the maximum one) for every stage $k$ and every player $i$, there is a subset of $\mathcal{K}_{i}$, call it $\mathcal{J}_{i}^{k}$, which is a partition of $T^{k}$. For every subset of players $J \subseteq N$, denote by $\mathcal{M}_{J}$ the meet (finest common coarsening) of $\left\{\mathcal{K}_{i}\right\}_{i \in J}$. Then for every stage $k$ there is a subset of $\mathcal{M}_{J}$, call it $\mathcal{M}_{J}^{k}$, which is a partition of $T^{k}$. If the information partition is the maximum one, namely $\left\langle\overline{\mathcal{K}}_{1}, \ldots, \overline{\mathcal{K}}_{n}\right\rangle$, then we shall denote the corresponding meets by $\overline{\mathcal{M}}_{J}$ and $\overline{\mathcal{M}}_{J}^{k}$, respectively. We want to provide an alternative characterization of $\overline{\mathcal{M}}_{J}^{k}$, that is, a characterization which is not in terms of the concept of "common coarsening" of the partitions $\left\{\overline{\mathcal{K}}_{i}^{k}\right\}_{i \in J}$. In order to do this we repeat the construction of Section 4 with respect to an arbitrary set of player $J \subseteq N$ (instead of a single player $i$ ).

For every node $t$ and for every set of players $J \subseteq N$, let $\mathcal{H}_{J}(t)$ be the set of information sets of players in $J$ that are crossed by paths starting at $t$ :

$$
\mathcal{H}_{J}(t)=\left\{h \in \bigcup_{i \in J} \mathbb{W}_{i} \mid \exists y \in h: t \precsim y\right\} .
$$

Next we introduce, for every $J \subseteq N$, a relation on $T$, denoted by $\sim_{J}$. Let $v, w \in T$ and $J \subseteq N$. Then $v \sim_{J} w$ if and only if, either (i) $v=w$, or (ii) $\ell(v)=\ell(w)$ and $\mathcal{H}_{J}(v) \cap \mathcal{H}_{J}(w) \neq \phi$. The relation $\sim_{J}$ is clearly reflexive and symmetric. However, in general, it is not transitive. Let $\sim_{J}^{*}$ denote the transitive closure of $\sim_{J}$. Thus $v \sim_{J}^{*} w$ if and only if there exists a finite sequence of nodes $\left\langle y_{1}, y_{2}, \ldots, y_{m}\right\rangle$ such that $y_{1}=v, y_{m}=w$ and, for all $k=1, \ldots, m-1, y_{k} \sim_{J} y_{k+1}$. Then $\sim_{J}^{*}$ is an equivalence relation on $T^{k}$.

Let

$$
H_{J}(t)=\left\{v \in T^{\ell(t)} \mid v \sim_{J}^{*} t\right\}
$$

and

$$
\mathbb{Q}_{J}^{k}=\left\{H_{J}(t) \mid t \in T^{k}\right\} .
$$

Proposition 4 shows that the equivalence relation $\sim_{J}^{*}$ represents, at each stage, the smallest event which is common knowledge among the players in $J$, under maximum information.

PROPOSITION 4: for every stage $k$ and every subset of players $J \subseteq N$, $\overline{\mathcal{M}}_{J}^{k}=\mathbb{U}_{J}^{k}$.

PROOF: in order to simplify the notation, we shall denote the cell of $\overline{\mathcal{M}}_{J}^{k}$ containing node $t$ as $\bar{M}_{J}(t)$. It is well-known that $v \in \bar{M}_{J}(t)$ if and only if there exists a finite sequence of nodes $\left\langle y_{1}, y_{2}, \ldots, y_{m}\right\rangle$ and a finite sequence of players $\left\langle i_{2}, \ldots, i_{m}\right\rangle$ in J, such that: $y_{1}=v, y_{m}=t$, and, for every $j=1, \ldots, m-1, y_{j} \in H_{i_{j+1}}\left(y_{j+1}\right)$ (see Figure H). We 


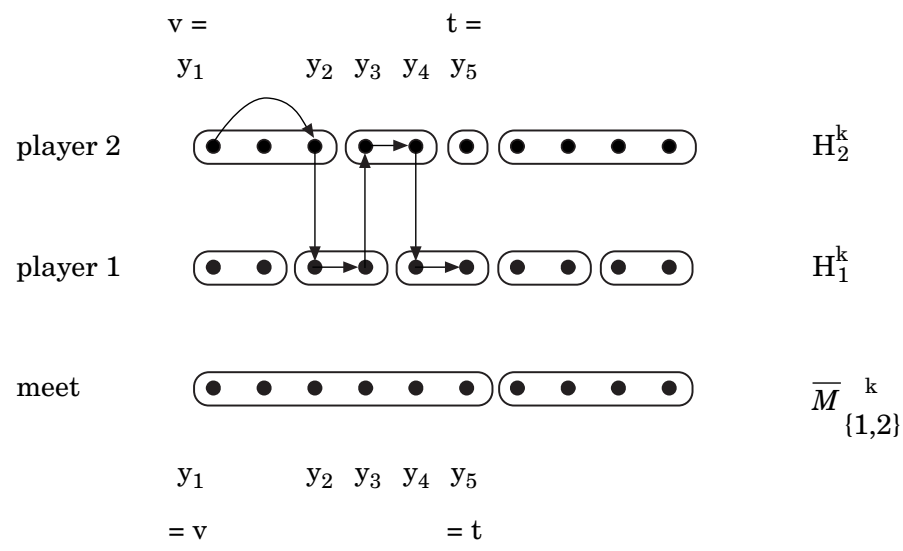

FIgURE H.

first show that $\bar{M}_{J}^{k}$ is (weakly) finer than $\mathbb{U}_{J}^{k}$, that is, $\forall t \in T^{k}$, $\bar{M}_{J}(t) \subseteq H_{J}(t)$. Let $v \in \bar{M}_{J}(t)$ and let $\left\langle y_{1}, y_{2}, \ldots, y_{m}\right\rangle$ and $\left\langle i_{2}, \ldots, i_{m}\right\rangle$ be sequences (of nodes and players, respectively) with the abovestated property. Since $y_{j} \in H_{i_{j+1}}\left(y_{j+1}\right)$, there is a sequence of nodes $\left\langle x_{j_{1}}, \ldots, x_{j_{m(j)}}\right\rangle$ in $T^{k}$ (which, w.l.o.g., we can assume to be distinct) such that, for all $r=1, \ldots, m(j)-1, \phi \neq \mathcal{H}_{i_{j+1}}\left(x_{j_{r}}\right) \cap H_{i_{j+1}}\left(x_{j_{r+1}}\right), x_{j_{1}}=$ $y_{j}, x_{j_{m(j)}}=y_{j+1}$. Since $\forall i_{j+1}, \forall w, \mathcal{H}_{i_{j+1}}(w) \subseteq \mathcal{H}_{J}(w)$, the sequence $v=y_{1}=x_{1_{1}}, \ldots, x_{1_{m(1)}}=y_{2}=x_{2_{1}}, \ldots, x_{2_{m(2)}}=y_{3}, \ldots, y_{m}=t$ is such that each pair of consecutive elements is $\sim_{J}$-related. Therefore, $v \sim_{J}^{*} t$, or $v \in H_{J}(t)$.

Now we show that $\mathbb{U}_{J}^{k}$ is (weakly) finer than $\overline{\mathcal{M}}_{J}^{k}$, that is, $\forall t \in T^{k}$, $H_{J}(t) \subseteq \bar{M}_{J}(t)$. Let $v \in H_{J}(t)$. Then there exist sequences $\left\langle t_{1}, \ldots, t_{m}\right\rangle$ and $\left\langle h_{2}, \ldots, h_{m}\right\rangle$ of nodes and information sets, respectively, such that, $t_{1}=v, t_{m}=t$, (w.l.o.g.) $t_{j} \neq t_{j+1}$, for all $j=1, \ldots, m, t_{j} \in T^{k}$, and, for all $j=1, \ldots, m-1, h_{j+1} \in \mathcal{H}_{J}\left(t_{j}\right) \cap \mathcal{H}_{J}\left(t_{j+1}\right)$. Let $i_{j+1} \in J$ be the player moving at $h_{j+1}$. Then $h_{j+1} \in \mathcal{H}_{i_{j+1}}\left(t_{j}\right) \cap \mathcal{H}_{i_{j+1}}\left(t_{j+1}\right)$, which implies that $t_{j} \sim_{i_{j+1}} t_{j+1}$. Therefore the sequences $\left\langle t_{1}, t_{2}, \ldots, t_{m}\right\rangle$ and $\left\langle i_{2}, \ldots, i_{m}\right\rangle$ (of nodes and players, respectively) are such that $\left\{i_{2}, \ldots, i_{m}\right\} \subseteq J, t_{1}=v, t_{m}=t$, and, for all $j=1, \ldots, m-1$, $t_{j} \in H_{i_{j+1}}\left(t_{j+1}\right)$, that is, $v \in \bar{M}_{J}(t)$.

EXAMPLE 3: consider the game of Figure A. Let us focus on stage 2 . In order to find the meet $\overline{\mathcal{M}}_{\{1,2,3\}}^{2}$ of the stage-2 maximum information partitions $\overline{\mathcal{K}}_{1}^{2}, \overline{\mathcal{K}}_{2}^{2}$ and $\overline{\mathcal{K}}_{3}^{2}$ we can either compute them first and then calculate their meet (as we did in Section 4) or - using proposition 4 -we can compute it directly using the relation $\sim_{J}^{*}$ defined above for $J=N=\{1,2,3\}$. This is quickly done as follows: $t_{2}, t_{3}$ and $t_{4}$ must all belong to the same cell, since they belong to an information set of one of the players, namely player 2 . Furthermore, $t_{4}$ and $t_{6}$ must belong to the same cell since there is 
an information set of one of the players, namely player 3 , that is crossed by a path starting at $t_{4}$ and also by a path starting at $t_{6}$. On the other hand, since $t_{7}$ is a terminal node, its cell is the singleton $\left\{t_{7}\right\}$. Thus $\overline{\mathcal{M}}_{\{1,2,3\}}^{2}=\left\{\left\{t_{2}, t_{3}, t_{4}, t_{6}\right\},\left\{t_{7}\right\}\right\}$.

\section{Final remarks and conclusion}

Restricting attention to the class of extensive forms defined by von Neumann and Morgenstern (1944) with the added assumption of perfect recall, we specified the information of each player at each node of the game-tree in a way which is coherent with the original information structure of the extensive form. We showed that this approach provides a framework for a formal and rigorous treatment of questions of knowledge and common knowledge at every node of the tree. We constructed a particular information completion and showed that it captures the notion of maximum information in the sense that it is the finest within the class of information completions that satisfy four natural properties. Using this notion of "maximum information" we were able to provide an alternative characterization of the meet of the information partitions. We also showed that, with maximum information, it is indeed true (as normally argued at an informal level) that at any node in a subgame it is common knowledge among all the players that the play of the game has reached that subgame. However, in general-that is, with less than maximum information-this statement is false (as a matter of fact the weaker statement that "all players know that the subgame has been reached" is false in general). On the other hand it is always true (that is, for arbitrary information completions) that in simultaneous games at every decision node there are no non-trivial events that are common knowledge among all the players.

Throughout the paper attention was restricted to $\mathrm{vN}-\mathrm{M}$ extensive forms. The reason for this is that in games that do not have this multi-stage structure, it may be problematical to define, for every player, a partition of the set of nodes that satisfies the four natural properties of Section 3. Consider, for example, the extensive form of Figure I. Suppose that we want to construct an information partition for player 2 . If we want the partition to satisfy property (1) (coherence with the information structure) then it must be that $\left[x_{1}\right]_{2}=\left[x_{2}\right]_{2}=\left\{x_{1}, x_{2}\right\}$ and $\left[x_{4}\right]_{2}=\left[x_{5}\right]_{2}=\left\{x_{4}, x_{5}\right\}$. By property (2) (players remember their own past choices), $x_{0} \notin\left[x_{3}\right]_{2}$. But this means that $\left[x_{3}\right]_{2}=\left\{x_{3}\right\}$, that is, if node $x_{3}$ is reached, player 2 is informed. Then she will be able to discriminate between $x_{4}$ and $x_{5}$ depending on whether or not she received the information $\left\{x_{3}\right\}$. But this goes against the notion of information set. Alternatively, one could weaken property (1) from equality to weak inclusion: 


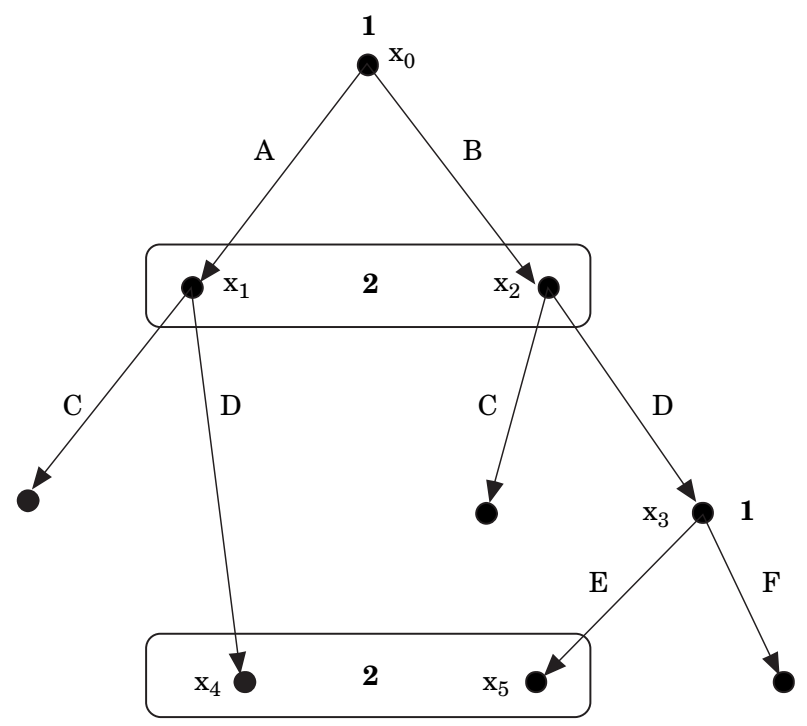

Figure I.

if $t \in h \in \mathbb{H}_{i}$ then $[t]_{i} \supseteq h$. Following this route one would have to choose between $x_{3} \in\left[x_{1}\right]_{2}$ and $x_{3} \in\left[x_{4}\right]_{2}$. In the former case property (2) would be violated (players remember their own past choices). In the latter case player 2 also needs to be told whether or not she has to move. If she is told that it is not her turn to move, then she will be able to deduce that node $x_{3}$ has been reached, leading to her ability to distinguish between $x_{4}$ and $x_{5}$ later on. All these problems can be eliminated by adding a dummy node and a dummy player with only one choice half way along the arc from $x_{1}$ to $x_{4}$, that is, by transforming the game into a vN-M one.

Prompted by a referee we conclude by discussing the connection between our notion of extended information structure and the notion of "normal form information set" introduced by Mailath, Samuelson and Swinkels (1993, 1994) (from now on MSS). Every information set $h$ for any player $i$ in a perfect recall game corresponds to the Cartesian product $S_{i}(h) \times S_{-i}(h)$ of the strategy profiles of the reduced normal form inducing a path through $h$. MSS identify the crucial structural property of such sets and propose to consider any set of strategy profiles of a reduced normal form game satisfying this property as a normal form information set. Of course, in general not all the normal form information sets of a given player correspond to actual information sets of the original extensive form. For example, the whole set of strategy profiles of the reduced normal form is a normal form information set for every player, but only one player moves at the root of the original extensive game. Analogously, we define sets of nodes which represent the information of a given player at any point of an extensive game and 
need not correspond to his actual information sets. For example, the singleton containing the root of the game is an "extended information set" for every player, although only one moves at the root. The obvious question is then: What is the relationship between extended information sets and normal form information sets? The answer is simple and does not depend on the precise mathematical definition given by MSS: an extended information set for player $i$ always corresponds to a normal form information for $i$ in the reduced normal form of the extensive game. In fact, let $t$ be a node at which player $i$ does not move and consider the extended information set $H_{i}(t)$. Now construct a modified extensive game in which an additional stage is added, just before the stage $\ell(t)$ containing $t$. The nodes in this stage are just a copy of those in $\ell(t)$, but formally are decision nodes of player $i$. However, player $i$ has a unique action at every such node leading to the corresponding node in stage $\ell(t)$ and his information sets are a copy of $i$ 's extended information sets in stage $\ell(t)$. It is obvious from this construction that the modified game must have the same reduced normal form as the original one and that the strategy profiles inducing a path through $H_{i}(t)$ are precisely those which induce a path through the information set of the modified game containing the copy of node $t$. Since this is a proper information set (although a trivial one), the corresponding set of strategy profiles must be a normal form information set. (However, since player $i$ does not move at $t$, the normal form information set corresponding to $H_{i}(t)$ is not "strict" in the sense of MSS, 1994: definition 8.) On the other hand, it is easy to produce examples of normal form information sets which do not correspond to any extended information set of the original extensive form game (see, for example, MSS, 1994: figures 1 and 2).

\section{Acknowledgements}

We thank Kluwer Academic Publishers for allowing publication of this paper in Research in Economics to improve the typesetting of the mathematical notation. The paper was first published in M.O.L. Bacharach, L.-A. Gérard-Varet, P. Mongin \& H.S. Shin, Eds. Epistemic Logic and the Theory of Games and Decisions. Kluwer Academic Publishers, 1997.

\section{References}

Aumann, R. (1976). Agreeing to disagree. Annals of Statistics, 4, 1236-1239.

Aumann, R. \& Brandenburger, A. (1995). Epistemic conditions for Nash equilibrium. Econometrica, 63, 1161-1180.

Aumann, R. (1995). Backward induction and common knowledge of rationality. Games and Economic Behaviour, 8, 6-19. 
Bacharach, M. (1985). On a claim of Aumann in an axiomatic model of knowledge. Journal of Economic Theory, 37, 167-190.

Battigalli, P. (1987). Comportamento razionale ed equilibrio nei giochi e nelle situazioni sociali. Unpublished dissertation, Università Commerciale L. Bocconi, Milan.

Battigalli, P. \& Guaitoli, P. (1997). Conjectural equilibria and rationalisability in a macroeconomic game with incomplete information. In P. Battigalli, A. Montesano \& F. Panunzi, Eds. Decisions, Games and Markets. Kluwer Academic Publishers.

Benoit, J.-P. \& Krishna, V. (1993). Renegotiation in finitely repeated games. Econometrica, 61, 303-323.

Bernheim, D., Peleg, B. \& Whinston, M. (1987). Coalition-proof Nash equilibria. I: Concepts. Journal of Economic Theory, 42, 1-12.

Bonanno, G. (1992a). Players' information in extensive games. Mathematical Social Sciences, 24, 35-48.

Bonanno, G. (1992b). Rational beliefs in extensive games. Theory and Decision, 33, 153-176.

Bonanno, G. (1992c). Set-theoretic equivalence of extensive-form games. International Journal of Game Theory, 20, 429-447.

Bonanno, G. (1994). Rationally acceptable recommendations in extensive games. University of California Davis, Mimeo.

Bonanno, G. (1995). A characterisation of sequential equilibrium. Economic Notes, 24, 109-128.

Bonanno, G. (1996). On the logic of common belief. Mathematical Logic Quarterly, 42, 305-311.

Farrell, J. \& Maskin, E. (1989). Renegotiation in repeated games. Games and Economic Behavior, 1, 327-360.

Fudenberg, D. \& Levine, D. (1993). Self-confirming equilibrium. Econometrica, 61, 523-545.

Geanakoplos, J. (1992). Common knowledge. Journal of Economic Perspectives, 6, 53-82.

Greenberg, J. (1990). The Theory of Social Situations. Cambridge: Cambridge University Press.

Grossman, S. \& Perry, M. (1986). Perfect sequential equilibrium. Journal of Economic Theory, 39, 97-119.

Halpern, J. (1986). Reasoning about knowledge: an overview. In J. Halpern, Ed. Theoretical Aspects of Reasoning about Knowledge. pp. 1-17. Los Altos, CA: Morgan Kaufmann.

Halpern, J. \& Moses, Y. (1992). A guide to completeness and complexity for modal logics of knowledge and belief. Artificial Intelligence, 54, 319-379.

Kalai, E. \& Lehrer, E. (1993a). Subjective equilibrium in repeated games. Econometrica, 61, 1231-1240.

Kalai, E. \& Lehrer, E. (1993b). Rational learning leads to Nash equilibrium. Econometrica, 61, 1019-1045.

Lismont, L. (1993). La connaissance commune en logique modale. Mathematical Logic Quarterly, 39, 115-130.

Lismont, L. \& Mongin, P. (1994). On the logic of common belief and common knowledge. Theory and Decision, 37(1), 75-106.

Mailath, G., Samuelson, L. \& Swinkels, J. (1993). Extensive form reasoning in normal form games. Econometrica, 61, 273-302.

Mailath, G., Samuelson, L. \& Swinkels, J. (1994). Normal form structures in extensive form games. Journal of Economic Theory, 64, 325-371.

Maskin, E. \& Tirole, J. (1994). Markov perfect equilibria. Harvard University, Mimeo.

Milgrom, P. (1981). An axiomatic characterisation of common knowledge. Econometrica, 49, 219-222. 
Noeldeke, G. \& van Damme, E. (1990). Switching away from probability one beliefs. Discussion Paper A-304, University of Bonn.

Rubinstein, A. \& Wolinsky, A. (1994). Rationalisable conjectural equilibrium: between Nash and rationalisability. Games and Economic Behavior, 6, 299-311.

Selten, R. (1975). Re-examination of the perfectness concept in extensive games. International Journal of Game Theory, 4, 25-55.

Thompson, F. (1952). Equivalence of games in extensive form. Research Memorandum No. 759, The Rand Corporation.

von Neumann, J. \& Morgenstern, O. (1944). Theory of Games and Economic Behavior. Princeton University Press.

\section{Appendix}

The notation and terminology used in this paper are quite standard. For the reader's convenience we provide a list below.

\begin{tabular}{|c|c|}
\hline Notation & Terminology \\
\hline$N=\{1$ & $\begin{array}{l}\text { Set of personal players (that is, excluding } \\
\text { Nature) }\end{array}$ \\
\hline$T$ & Set of nodes \\
\hline$Z$ & Set of terminal nodes \\
\hline$\prec$ & $\begin{array}{l}\text { Strict precedence relation on } T: t \prec t^{\prime} \text { if and } \\
\text { only if } t \neq t^{\prime} \text { and } t \text { is a predecessor of } t^{\prime}\end{array}$ \\
\hline$\precsim$ & $\begin{array}{l}\text { Weak precedence relation on } T: t \precsim t^{\prime} \text { if either } \\
t=t^{\prime} \text { or } t \prec t^{\prime}\end{array}$ \\
\hline$t_{0}$ & Root of the tree \\
\hline$p(t)$ & Immediate predecessor of node $t\left(t \in T \backslash\left\{t_{0}\right\}\right)$ \\
\hline $\mathcal{S}(t)$ & Set of immediate successors of node $t$ \\
\hline $\mathcal{S}(h, c)$ & $\begin{array}{l}\text { Set of immediate successors of nodes in } \\
\text { information set } h \text { following the edges that } \\
\text { constitute choice } c(\text { at } h \text { ) }\end{array}$ \\
\hline$\ell(t)$ & $\begin{array}{l}\text { Number of predecessors of node } t \text { (thus } \\
\ell\left(t_{0}\right)=0 \text { and, for every } t \neq t_{0} \\
\ell(t)=\ell(p(t))+1)\end{array}$ \\
\hline$T^{k}(k \in \mathbb{N})$ & $\begin{array}{l}\text { Set of "stage- } k \text { (or layer- } k \text { ) nodes": } \\
T^{k}=\{t \in T: \ell(t)=k\}\end{array}$ \\
\hline $\mathfrak{H}_{i}(i \in$ & Set of player $i$ 's information sets \\
\hline$H(t)(t \in T \backslash Z)$ & Information set to which node $t$ belongs \\
\hline
\end{tabular}

